The Journal of $\mathbf{N}_{\text {onlinear }} \mathbf{S}$ ciences and Applications http://www.tjnsa.com

\title{
HYERS-ULAM-RASSIAS STABILITY OF THE APOLLONIUS TYPE QUADRATIC MAPPING IN RN-SPACES
}

\author{
H. AZADI KENARY ${ }^{1, *}$, KH. SHAFAAT ${ }^{2}$, M. SHAFEI ${ }^{3}$ AND G. TAKBIRI ${ }^{4}$ \\ Dedicated to Professor Themistocles M. Rassias on the occasion of his sixtieth birthday
}

Abstract. Recently, in [5], Najati and Moradlou proved Hyers-Ulam-Rassias stability of the following quadratic mapping of Apollonius type

$$
Q(z-x)+Q(z-y)=\frac{1}{2} Q(x-y)+2 Q\left(z-\frac{x+y}{2}\right)
$$

in non-Archimedean space. In this paper we establish Hyers-Ulam-Rassias stability of this functional equation in random normed spaces by direct method and fixed point method. The concept of Hyers-Ulam-Rassias stability originated from Th. M. Rassias stability theorem that appeared in his paper: On the stability of the linear mapping in Banach spaces, Proc. Amer. Math. Soc. 72 (1978), 297-300.

\section{INTRODUCTION AND PRELIMINARIES}

The stability problem of functional equations originated from a question of Ulam [10] concerning the stability of group homomorphisms. Hyers [4] gave a first affirmative partial answer to the question of Ulam for Banach spaces. Hyers's theorem was generalized by Th. M. Rassias [6] for linear mappings by considering an unbounded Cauchy difference.

Theorem 1.1. let $f$ be an approximately additive mapping from a normed vector space $E$ into a Banach space $E^{\prime}$, i.e., $f$ satisfies the inequality

$$
\|f(x+y)-f(x)-f(y)\| \leq \epsilon\left(\|x\|^{r}+\|y\|^{r}\right)
$$

Date: Received: September 29, 2010; Revised: November 23, 2010.

(C) 2010 N.A.G

${ }^{*}$ Corresponding author.

2000 Mathematics Subject Classification. Primary 39B22, 39B52, 39B82, 46 S10.

Key words and phrases. Fixed point theory; Stability; Random normed space. 
for all $x, y \in E$, where $\epsilon$ and $r$ are constants with $\epsilon>0$ and $0 \leq r<1$. Then the mapping $L: E \rightarrow E^{\prime}$ defined by $L(x)=\lim _{n \rightarrow \infty} 2^{-n} f\left(2^{n} x\right)$ is the unique additive mapping which satisfies

for all $x \in E$.

$$
\|f(x+y)-L(x)\| \leq \frac{2 \epsilon}{2-2^{r}}\|x\|^{r}
$$

The paper of Th.M. Rassias [6] has provided a lot of influence in the development of what we call generalized Hyers-Ulam stability or Hyers-Ulam-Rassias stability of functional equations. A generalization of the Th.M. Rassias theorem was obtained by Găvruta [3] by replacing the unbounded Cauchy difference by a general control function in the spirit of Th. M. Rassias approach.

The functional equation

$$
f(x+y)+f(x-y)=2 f(x)+2 f(y),
$$

is called a quadratic functional equation. In particular, every solution of the quadratic functional equation is said to be a quadratic mapping. The HyersUlam-Rassias stability problem for the quadratic functional equation was proved by Skof [9] for mappings $f: X \rightarrow Y$, where $X$ is a normed space and $Y$ is a Banach space. Cholewa [1] noticed that the theorem of Skof is still true if the relevant domain $X$ is replaced by an Abelian group. Czerwik [2] proved the Hyers-Ulam-Rassias stability of the quadratic functional equation.

A. Najati and F. Moradlou [5], introduced the following functional equation

$$
Q(z-x)+Q(z-y)=\frac{1}{2} Q(x-y)+2 Q\left(z-\frac{x+y}{2}\right)
$$

and he established the general solution and the generalized Hyers-Ulam- Rassias stability problem for the functional equation (1.2) in non-Archimedean spaces. In this paper, we establish the Hyers-Ulam-Rassias stability problem of functional equation (1.2) in random normed spaces.

In the sequel, we shall adopt the usual terminology, notions and conventions of the theory of random normed spaces as in [8]. Throughout this paper, the spaces of all probability distribution functions is denoted by $\triangle^{+}$. Elements of $\triangle^{+}$are functions $F: \mathbb{R} \cup[-\infty,+\infty] \rightarrow[0,1]$, such that $F$ is left continuous and nondecreasing on $\mathbb{R}$ and $F(0)=0, F(+\infty)=1$. It's clear that the subset

$$
D^{+}=\left\{F \in \triangle^{+}: l^{-} F(-\infty)=1\right\},
$$

where $l^{-} f(x)=\lim _{t \rightarrow x^{-}} f(t)$, is a subset of $\Delta^{+}$. The space $\Delta^{+}$is partially ordered by the usual pointwise ordering of functions, that is for all $t \in \mathbb{R}, F \leq G$ if and only if $F(t) \leq G(t)$. For every $a \geq 0, H_{a}(t)$ is the element of $D^{+}$defined by

$$
H_{a}(t)=\left\{\begin{array}{ll}
0 & \text { if } t \leq a \\
1 & \text { if } t>a
\end{array} .\right.
$$

One can easily show that the maximal element for $\Delta^{+}$in this order is the distribution function $H_{0}(t)$.

Definition 1.2. A function $T:[0,1]^{2} \rightarrow[0,1]$ is a continuous triangular norm (briefly a $t$-norm) if $T$ satisfies the following conditions: 
(1) $T$ is commutative and associative;

(2) $T$ is continuous;

(3) $T(x, 1)=x$ for all $x \in[0,1]$;

(4) $T(x, y) \leq T(z, w)$ whenever $x \leq z$ and $y \leq w$ for all $x, y, z, w \in[0,1]$.

Three typical examples of continuous $t$-norms are $T(x, y)=x y, T(x, y)=$ $\max \{a+b-1,0\}$ and $T(x, y)=\min (a, b)$. Recall that, if $T$ is a $t$-norm and $\left\{x_{n}\right\}$ is a given of numbers in $[0,1], T_{i=1}^{n} x_{i}$ is defined recursively by $T_{i=1}^{1} x_{1}$ and $T_{i=1}^{n} x_{i}=T\left(T_{i=1}^{n-1} x_{i}, x_{n}\right)$ for $n \geq 2$.

Definition 1.3. A random normed space(briefly $R N$-space) is a triple $(X, \Psi, T)$, where $X$ is a vector space, $T$ is a continuous $t$-norm and $\Psi: X \rightarrow D^{+}$is a mapping such that the following conditions hold:

(1) $\Psi_{x}(t)=H_{0}(t)$ for all $t>0$ if and only if $x=0$.

(2) $\Psi_{\alpha x}(t)=\Psi_{x}\left(\frac{t}{|\alpha|}\right)$ for all $\alpha \in \mathbb{R}, \alpha \neq 0, x \in X$ and $t \geq 0$.

(3) $\Psi_{x+y}(t+s) \geq T\left(\Psi_{x}(t), \Psi_{y}(s)\right)$, for all $x, y \in X$ and $t, s \geq 0$.

Every normed space $(X,\|\|$.$) defines a random normed space \left(X, \Psi, T_{M}\right)$ where for every $t>0$,

$$
\Psi_{u}(t)=\frac{t}{t+\|u\|}
$$

and $T_{M}$ is the minimum $t$-norm. This space is called the induced random normed space.

Definition 1.4. Let $(X, \Psi, T)$ be an RN-space.

(1) A sequence $\left\{x_{n}\right\}$ in $X$ is said to be convergent to $x \in X$ in $X$ if for all $t>0, \lim _{n \rightarrow \infty} \Psi_{x_{n}-x}(t)=1$.

(2) A sequence $\left\{x_{n}\right\}$ in $X$ is said to be Cauchy sequence in $X$ if for all $t>0$, $\lim _{n \rightarrow \infty} \Psi_{x_{n}-x_{m}}(t)=1$.

(3) The $R N$-space $(X, \Psi, T)$ is said to be complete if every Cauchy sequence in $X$ is convergent.

Theorem 1.5. If $(X, \Psi, T)$ is $R N$-space and $\left\{x_{n}\right\}$ is a sequence such that $x_{n} \rightarrow x$, then $\lim _{n \rightarrow \infty} \Psi_{x_{n}}(t)=\Psi_{x}(t)$.

Definition 1.6. Let $X$ be a set. A function $d: X \times X \rightarrow[0, \infty]$ is called a generalized metric on $X$ if $d$ satisfies

(1) $d(x, y)=0$ if and only if $x=y$,

(2) $d(x, y)=d(y, x)$ for all $x, y \in X$,

(3) $d(x, z) \leq d(x, y)+d(y, z)$ for all $x, y, z \in X$.

Theorem 1.7. Let $(X, d)$ be a complete generalized metric space and let $J: X \rightarrow$ $X$ be a strictly contractive mapping with Lipschitz constant $L<1$. Then for each given element $x \in X$, either

$$
d\left(J^{n} x, J^{n+1} x\right)=\infty
$$

for all nonnegative integers $n$ or there exists a positive integer $n_{0}$ such that 
(1) $d\left(J^{n} x, J^{n+1} x\right)<\infty$ for all $n_{0} \geq n_{0}$;

(2) the sequence $\left\{J^{n} x\right\}$ converges to a fixed point $y^{*}$ of $J$;

(3) $y^{*}$ is the unique fixed point of $J$ in the set $Y=\left\{y \in X \mid d\left(J^{n_{0}} x, y\right)<\infty\right\}$;

(4) $d\left(y, y^{*}\right) \leq \frac{1}{1-L} d(y, J y)$ for all $y \in Y$.

\section{RN-stability of the functional equation (1.2): direct method}

In this section, we use direct method to prove the Hyres-Ulam-Rassias stability of quadratic functional equations (1.2).

Theorem 2.1. Let $X$ be a real linear space, $(Z, \Psi, \min )$ be an $R N$-space, $\zeta$ : $X^{3} \rightarrow Z$ be a function such that for some $0<\alpha<4$,

$$
\Psi_{\zeta(2 x, 2 y, 2 z)}(t) \geq \Psi_{\alpha \zeta(x, y, z)}(t) \quad \forall x \in X, t>0,
$$

$Q(0)=0$ and for all $x, y, z \in X$ and $t>0$

$$
\lim _{n \rightarrow \infty} \Psi_{\zeta\left(2^{n} x, 2^{n} y, 2^{n} z\right)}\left(4^{n} t\right)=1 .
$$

Let $(Y, \mu$, min $)$ be a complete $R N$-space. If $Q: X \rightarrow Y$ is a mapping such that for all $x, y, z \in X$ and $t>0$,

$$
\mu_{Q(z-x)+Q(z-y)-\frac{1}{2} Q(x-y)-2 Q\left(z-\frac{x+y}{2}\right)}(t) \geq \Psi_{\zeta(x, y, z)}(t),
$$

then there is a unique quadratic mapping $R: X \rightarrow Y$ such that

$$
R(x):=\lim _{n \rightarrow \infty} 4^{-n} Q\left(2^{n} x\right),
$$

and

$$
\mu_{Q(x)-R(x)}(t) \geq \Psi_{\zeta(x,-x, x)}\left(\frac{(4-\alpha) t}{2}\right) .
$$

Proof. Putting $y=-x$ and $z=x$ in (2.2) we see that for all $x \in X$ and all $t>0$,

$$
\mu_{\frac{Q(2 x)}{4}-Q(x)}(t) \geq \Psi_{\zeta(x,-x, x)}(2 t)
$$

Replacing $x$ by $2^{n} x$ in (2.4) and using (2.1), we obtain

$$
\mu_{\frac{Q\left(2^{n+1} x\right)}{4^{n+1}}-\frac{Q\left(2^{n} x\right)}{4^{n}}}(t) \geq \Psi_{\psi\left(2^{n} x,-2^{n} x, 2^{n} x\right)}\left(2 \times 4^{n} t\right) \geq \Psi_{\zeta(x,-x, x)}\left(\frac{2 \times 4^{n} t}{\alpha^{n}}\right) .
$$

So

$$
\begin{aligned}
\mu_{\frac{Q\left(2^{n} x\right)}{4^{n}}-Q(x)}\left(\sum_{k=0}^{n-1} \frac{t \alpha^{k}}{2 \times 4^{k}}\right) & =\mu_{\sum_{k=0}^{n-1} \frac{Q\left(2^{k+1} x\right)}{4^{k+1}}-\frac{Q\left(2^{k} x\right)}{4^{k}}}\left(\sum_{k=0}^{n-1} \frac{t \alpha^{k}}{2 \times 4^{k}}\right) \\
& \geq T_{k=0}^{n-1} \mu_{\frac{Q\left(2^{k+1} x\right)}{4^{k+1}}-\frac{Q\left(2^{k} x\right)}{4^{k}}}\left(\frac{t \alpha^{k}}{2 \times 4^{k}}\right) \\
& \geq T_{k=0}^{n-1}\left(\Psi_{\zeta(x,-x, x)}(t)\right) \\
& =\Psi_{\zeta(x,-x, x)}(t) .
\end{aligned}
$$

This implies that

$$
\mu_{\frac{Q\left(2^{n} x\right)}{4^{n}}-Q(x)}(t) \geq \Psi_{\psi(x,-x, x)}\left(\frac{t}{\sum_{k=0}^{n-1} \frac{\alpha^{k}}{2 \times 4^{k}}}\right) .
$$


Replacing $x$ by $2^{p} x$ in (2.6), we obtain

$$
\begin{aligned}
\mu_{\frac{Q\left(2^{n+p} x\right)}{4^{n+p}}-\frac{Q\left(2^{p} x\right)}{4^{p}}}(t) & \geq \Psi_{\zeta\left(2^{p} x,-2^{p} x, 2^{p} x\right)}\left(\frac{t}{\sum_{k=0}^{n-1} \frac{\alpha^{k}}{2 \times 4^{k+p}}}\right) \\
& \geq \Psi_{\psi(x,-x, x)}\left(\frac{t}{\sum_{k=0}^{n-1} \frac{\alpha^{k+p}}{2 \times 4^{k+p}}}\right) \\
& =\Psi_{\zeta(x,-x, x)}\left(\frac{t}{\sum_{k=p}^{n+p-1} \frac{\alpha^{k}}{2 \times 4^{k}}}\right) .
\end{aligned}
$$

As

$$
\lim _{p, n \rightarrow \infty} \Psi_{\zeta(x,-x, x)}\left(\frac{t}{\sum_{k=p}^{n+p-1} \frac{\alpha^{k}}{2 \times 4^{k}}}\right)=1,
$$

then $\left\{\frac{Q\left(2^{n} x\right)}{4^{n}}\right\}_{n=1}^{\infty}$ is a Cauchy sequence in complete RN-space $(Y, \mu, \min )$, so there exist some point $R(x) \in Y$ such that

$$
\lim _{n \rightarrow \infty} 4^{-n} Q\left(2^{n} x\right)=R(x) .
$$

Fix $x \in X$ and put $p=0$ in (2.8). Then we obtain

$$
\mu_{\frac{Q\left(2^{n} x\right)}{4^{n}}-Q(x)}(t) \geq \Psi_{\zeta(x,-x, x)}\left(\frac{t}{\sum_{k=0}^{n-1} \frac{\alpha^{k}}{2 \times 4^{k}}}\right),
$$

and so, for every $\epsilon>0$, we have

$$
\begin{aligned}
\mu_{R(x)-Q(x)}(t+\epsilon) & \geq T\left(\mu_{R(x)-\frac{Q\left(2^{n} x\right)}{4^{n}}}(\epsilon), \mu_{\frac{Q\left(2^{n} x\right)}{4^{n}}-Q(x)}(t)\right) \\
& \geq T\left(\mu_{R(x)-\frac{Q\left(2^{n}\right)}{4^{n}}}(\epsilon), \Psi_{\zeta(x,-x, x)}\left(\frac{t}{\sum_{k=0}^{n-1} \frac{\alpha^{k}}{2 \times 4^{k}}}\right)\right) .
\end{aligned}
$$

Taking the limit as $n \rightarrow \infty$ and using (2.10), we get

$$
\mu_{R(x)-Q(x)}(t+\epsilon) \geq \Psi_{\psi(x,-x, x)}\left(\frac{(4-\alpha) t}{2}\right) .
$$

Since $\epsilon$ was arbitrary by taking $\epsilon \rightarrow 0$ in (2.11), we get

$$
\mu_{R(x)-Q(x)}(t) \geq \Psi_{\zeta(x,-x, x)}\left(\frac{(4-\alpha) t}{2}\right)
$$

Replacing $x, y$ and $z$ by $2^{n} x, 2^{n} y$ and $2^{n} z$, respectively, in (2.2), we get for all $x, y, z \in X$ and for all $t>0$,

$$
\mu_{\frac{1}{4^{n}}\left[Q\left(2^{n} z-2^{n} x\right)+Q\left(2^{n} z-2^{n} y\right)-\frac{1}{2} Q\left(2^{n} x-2^{n} y\right)-2 Q\left(2^{n} z-\frac{x+y}{2^{n-1}}\right)\right]}(t) \geq \Psi_{\zeta\left(2^{n} x, 2^{n} y, 2^{n} z\right)}\left(4^{n} t\right) .
$$

Since $\lim _{n \rightarrow \infty} \Psi_{\zeta\left(2^{n} x, 2^{n} y, 2^{n} z\right)}\left(4^{n} t\right)=1$, we conclude that

$$
R(z-x)+R(z-y)=\frac{1}{2} R(x-y)+2 R\left(z-\frac{x+y}{2}\right) .
$$

To prove the uniqueness of the quadratic mapping $R$, assume that there exist another quadratic mapping $S: X \rightarrow Y$ which satisfies (2.3). By induction one 
can easily show that for all $n \in \mathbb{N}$ and every $x \in X, R\left(2^{n} x\right)=4^{n} R(x)$ and $S\left(m^{n} x\right)=4^{n} S(x)$. So

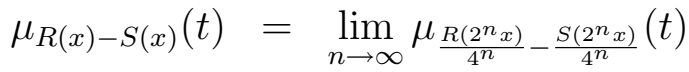

$$
\begin{aligned}
& \geq \lim _{n \rightarrow \infty} \min \left\{\mu_{\frac{R\left(2^{n} x\right)}{4^{n}}-\frac{Q\left(2^{n} x\right)}{4^{n}}}\left(\frac{t}{2}\right), \mu_{\frac{S\left(2^{n} x\right)}{4^{n}}-\frac{Q\left(2^{n} x\right)}{4^{n}}}\left(\frac{t}{2}\right)\right\} \\
& \geq \lim _{n \rightarrow \infty} \Psi_{\zeta\left(2^{n} x,-2^{n} x, 2^{n} x\right)}\left(\frac{4^{n}(4-\alpha) t}{4}\right) \\
& \geq \lim _{n \rightarrow \infty} \Psi_{\zeta(x,-x, x)}\left(\frac{4^{n}(4-\alpha) t}{4 \alpha^{n}}\right) .
\end{aligned}
$$

Since $\lim _{n \rightarrow \infty} \frac{4^{n}(4-\alpha) t}{4 \alpha^{n}}=\infty$, we get

$$
\lim _{n \rightarrow \infty} \Psi_{\zeta(x,-x, x)}\left(\frac{4^{n}(4-\alpha) t}{4 \alpha^{n}}\right)=1 .
$$

Therefore, it follows that for all $t>0, \mu_{R(x)-S(x)}(t)=1$ and so $R(x)=S(x)$. This complete the proof.

Corollary 2.2. Let $X$ be a real linear space, $(Z, \Psi, \min )$ be an $R N$-space, and $(Y, \mu$, min $)$ a complete $R N$-space. Let $0<p<1$ and $z_{0} \in Z$. If $Q: X \rightarrow Y$ is a mapping that for all $x, y \in X$ and $t>0$,

$$
\mu_{Q(z-x)+Q(z-y)-\frac{1}{2} Q(x-y)-2 Q\left(z-\frac{x+y}{2}\right)}(t) \geq \Psi_{\|y\|^{p} y_{0}}(t),
$$

then there is a unique quadratic mapping $R: X \rightarrow Y$ such that

$$
R(x)=\lim _{n \rightarrow \infty} 4^{-n} Q\left(2^{n} x\right),
$$

and

$$
\mu_{Q(x)-R(x)}(t) \geq \Psi_{\|x\|^{p} z_{0}}\left(\frac{\left(4-4^{p}\right) t}{2}\right) .
$$

Proof. Let $\alpha=4^{p}$ and $\zeta: X^{3} \rightarrow Z$ be defined by $\zeta(x, y, z)=\|y\|^{p} z_{0}$.

Corollary 2.3. Let $X$ be a real linear space, $(Z, \Psi$, min) be an $R N$-space, and $(Y, \mu, \min )$ a complete $R N$-space. Let $0<p<1$ and $z_{0} \in Z$. If $Q: X \rightarrow Y$ is a mapping that for all $x, y, z \in X$ and $t>0$,

$$
\mu_{Q(z-x)+Q(z-y)-\frac{1}{2} Q(x-y)-2 Q\left(z-\frac{x+y}{2}\right)}(t) \geq \Psi_{\left(\|x\|\left\|^{p}+\right\| y\|\|^{p}+\left.\|z\|\right|^{p}\right) z_{0}}(t),
$$

then there is a unique quadratic mapping $R: X \rightarrow Y$ such that

$$
R(x)=\lim _{n \rightarrow \infty} 4^{-n} Q\left(2^{n} x\right),
$$

and

$$
\mu_{Q(x)-R(x)}(t) \geq \Psi_{\|x\|^{p} z_{0}}\left(\frac{\left(4-4^{p}\right) t}{6}\right) .
$$

Proof. Let $\alpha=4^{p}$ and $\zeta: X^{3} \rightarrow Z$ be defined by $\zeta(x, y, z)=\left(\|x\|^{p}+\|y\|^{p}+\right.$ $\left.\| z||^{p}\right) z_{0}$ 
Corollary 2.4. Let $X$ be a real linear space, $(Z, \Psi, \min )$ be an $R N$-space, and $\left(Y, \mu\right.$, min) a complete $R N$-space. Let $z_{0} \in Z$. If $Q: X \rightarrow Y$ is a mapping that for all $x, y, z \in X$ and $t>0$,

$$
\mu_{Q(z-x)+Q(z-y)-\frac{1}{2} Q(x-y)-2 Q\left(z-\frac{x+y}{2}\right)}(t) \geq \Psi_{\delta z_{0}}(t),
$$

then there is a unique quadratic mapping $R: X \rightarrow Y$ such that for all $x \in X$ and $t>0$,

$$
R(x)=\lim _{n \rightarrow \infty} 4^{-n} Q\left(2^{n} x\right),
$$

and

$$
\mu_{Q(x)-R(x)}(t) \geq \Psi_{\delta z_{0}}\left(\frac{3 t}{2}\right) .
$$

Proof. Let $\alpha=1$ and $\psi: X^{3} \rightarrow Z$ be defined by $\psi(x, y)=\delta z_{0}$.

\section{RN-Stability of the functional equation (1.2): fixed point method}

Theorem 3.1. Let $X$ be a linear space, $\left(Y, \mu, T_{M}\right)$ be a complete $R N$-space and $\Lambda$ be a mapping from $X^{3}$ to $D^{+}\left(\Lambda(x, y, z)\right.$ is denoted by $\left.\Lambda_{x, y, z}\right)$ such that, for some $0<\alpha<\frac{1}{4}$,

$$
\Lambda_{2 x, 2 y, 2 z}(t) \leq \Lambda_{x, y, z}(\alpha t)
$$

for all $x, y, z \in X$ and all $t>0$. Let $Q: X \rightarrow Y$ be a quadratic mapping satisfying

$$
\mu_{Q(z-x)+Q(z-y)-\frac{1}{2} Q(x-y)-2 Q\left(z-\frac{x+y}{2}\right)}(t) \geq \Lambda_{x, y, z}(t)
$$

for all $x, y, z \in X$ and all $t>0$. Then

$$
R(x):=\lim _{n \rightarrow \infty} 4^{n} Q\left(\frac{x}{2^{n}}\right)
$$

exists for each $x \in X$ and defines a unique quadratic mapping $R: X \rightarrow Y$ such that

$$
\mu_{Q(x)-R(x)}(t) \geq \Lambda_{x,-x, x}\left(\frac{(1-4 \alpha) t}{2 \alpha}\right) .
$$

for all $x, y, z \in X$ and all $t>0$.

Proof. Putting $y=-x$ and $z=x$ in (3.2), we have

$$
\mu_{\frac{1}{2} Q(2 x)-2 Q(x)}(t) \geq \Lambda_{x,-x, x}(t),
$$

for all $x \in X$ and all $t>0$. Consider the set

$$
S:=\{g: X \rightarrow Y\}
$$

and introduce the generalized metric on $S$ :

$$
d(f, g)=\inf \left\{u \in \mathbb{R}^{+}: \mu_{g(x)-h(x)}(u t) \geq \Lambda_{x,-x, x}(t), \forall x \in X, \forall t>0\right\},
$$

where, as usual, inf $\emptyset=+\infty$. It is easy to show that $(S, d)$ is complete. Now we consider the linear mapping $J: S \rightarrow S$ such that

$$
J h(x):=4 h\left(\frac{x}{2}\right)
$$


for all $x \in X$ and we prove that $J$ is a strictly contractive mapping with the Lipschitz constant $4 \alpha$. Let $g, h \in S$ be given such that $d(g, h)<\epsilon$. Then

$$
\mu_{g(x)-h(x)}(\epsilon t) \geq \Lambda_{x,-x, x}(t)
$$

for all $x \in X$ and all $t>0$. Hence

$$
\begin{aligned}
\mu_{J g(x)-J h(x)}(4 \alpha \epsilon t) & =\mu_{4 g\left(\frac{x}{2}\right)-4 h\left(\frac{x}{2}\right)}(4 \alpha \epsilon t) \\
& =\mu_{g\left(\frac{x}{2}\right)-h\left(\frac{x}{2}\right)}(\alpha \epsilon t) \\
& \geq \Lambda_{\frac{x}{2},-\frac{x}{2}, \frac{x}{2}}(\alpha t) \\
& \geq \Lambda_{x,-x, x}(t)
\end{aligned}
$$

for all $x \in X$ and all $t>0$. So $d(g, h)<\epsilon$ implies that $d(J g, J h)<4 \alpha \epsilon$. This means that

$$
d(J g, J h) \leq 4 \alpha d(g, h)
$$

for all $g, h \in S$. It follows from (3.5) that

$$
\mu_{Q(x)-4 Q\left(\frac{x}{2}\right)}(\alpha t) \geq \Lambda_{x,-x, x}(t)
$$

for all $x \in X$ and all $t>0$. So

$$
d(Q, J Q) \leq 2 \alpha<1
$$

By Theorem (1.7), there exists a mapping $R: X \rightarrow Y$ satisfying the following:

(1) $R$ is a fixed point of $J$, that is

$$
R\left(\frac{x}{2}\right)=\frac{1}{4} R(x)
$$

for all $x \in X$. The mapping $R$ is a unique fixed point of $J$ in the set

$$
\Omega=\{h \in S: d(g, h)<\infty\} .
$$

This implies that $R$ is a unique mapping satisfying (3.14) such that there exists a $u \in(0, \infty)$ satisfying

$$
\mu_{Q(x)-R(x)}(u t) \geq \Lambda_{x,-x, x}(t)
$$

for all $x \in X$ and all $t>0$;

(2) $d\left(J^{n} Q, R\right) \rightarrow 0$ as $n \rightarrow \infty$. This implies the equality

$$
\lim _{n \rightarrow \infty} 4^{n} Q\left(\frac{x}{2^{n}}\right)=R(x)
$$

for all $x \in X$.

(3) $d(Q, R) \leq \frac{d(\dot{Q}, J Q)}{1-4 \alpha}$ with $Q \in \Omega$, which implies the inequality

$$
d(Q, R) \leq \frac{2 \alpha}{1-4 \alpha}
$$

from which it follows

$$
\mu_{Q(x)-R(x)}\left(\frac{2 \alpha t}{1-4 \alpha}\right) \geq \Lambda_{x,-x, x}(t)
$$


for all $x \in X$ and all $t>0$. This implies that the inequality (3.4) holds. Replacing $x, y$ and $z$ by $2^{n} x, 2^{n} y$ and $2^{n} z$, respectively in (3.2), we obtain

$$
\begin{aligned}
\mu_{4^{n}\left[Q\left(\frac{z}{2^{n}}-\frac{x}{2^{n}}\right)+Q\left(\frac{z}{2^{n}}-\frac{y}{2^{n}}\right)-\frac{1}{2} Q\left(\frac{x}{2^{n}}-\frac{y}{2^{n}}\right)-2 Q\left(\frac{z}{2^{n}}-\frac{x+y}{2^{n+1}}\right)\right]}(t) & \geq \Lambda_{\frac{x}{2^{n}}, \frac{y}{2^{n}}, \frac{z}{2^{n}}}\left(\frac{t}{4^{n}}\right) \\
& \geq \Lambda_{x, y, z}\left(\left(\frac{1}{4 \alpha}\right)^{n} t\right)
\end{aligned}
$$

for all $x, y, z \in X$, all $t>0$ and all $n \in \mathbb{N}$. Since $\lim _{n \rightarrow \infty} \Lambda_{x, y, z}\left(\left(\frac{1}{4 \alpha}\right)^{n} t\right)=1$ for all $x, y, z \in X$ and all $t>0$, then we deduce that

$$
\mu_{R(z-x)+R(z-y)-\frac{1}{2} R(x-y)-2 R\left(z-\frac{x+y}{2}\right)}(t)=1,
$$

for all $x, y, z \in X$ and all $t>0$. Thus the mapping $R: X \rightarrow Y$ is quadratic.

Corollary 3.2. Let $\theta \geq 0$ and let $p$ be a real number with $p>1$. Let $X$ be $a$ normed vector space with norm $\|$.$\| . Let Q: X \rightarrow Y$ be a mapping satisfying

$$
\mu_{Q(z-x)+Q(z-y)-\frac{1}{2} Q(x-y)-2 Q\left(z-\frac{x+y}{2}\right)}(t) \geq \frac{t}{t+\theta\left(\|x\|^{p}+\|y\|^{p}+\|z\| \|^{p}\right)}
$$

for all $x, y, z \in X$ and all $t>0$. Then

$$
R(x):=\lim _{n \rightarrow \infty} 4^{n} Q\left(\frac{x}{2^{n}}\right)
$$

exists for each $x \in X$ and defines a quadratic mapping $R: X \rightarrow Y$ such that

$$
\mu_{Q(x)-R(x)}(t) \geq \frac{\left(4^{p}-4\right) t}{\left(4^{p}-4\right) t+6 \theta\|x\|^{p}}
$$

Proof. The proof follows from Theorem (3.1) by taking

$$
\Lambda_{x, y, z}(t)=\frac{t}{t+\theta\left(\|x\|^{p}+\|y\|^{p}+\|z\|^{p}\right)}
$$

for all $x, y, z \in X$ and all $t>0$. Then we can choose $\alpha=4^{-p}$ and we get the desired result.

Similarly, we can obtain the following. We will omit the proof.

Theorem 3.3. Let $X$ be a linear space, $\left(Y, \mu, T_{M}\right)$ be a complete $R N$-space and $\Lambda$ be a mapping from $X^{3}$ to $D^{+}\left(\Lambda(x, y, z)\right.$ is denoted by $\left.\Lambda_{x, y, z}\right)$ such that, for some $0<\alpha<4$,

$$
\Lambda_{\frac{x}{2}, \frac{y}{2}, \frac{z}{2}}(t) \leq \Lambda_{x, y, z}(\alpha t)
$$

for all $x, y, z \in X$ and all $t>0$. Let $Q: X \rightarrow Y$ be a quadratic mapping satisfying

$$
\mu_{Q(z-x)+Q(z-y)-\frac{1}{2} Q(x-y)-2 Q\left(z-\frac{x+y}{2}\right)}(t) \geq \Lambda_{x, y, z}(t)
$$

for all $x, y, z \in X$ and all $t>0$. Then

$$
R(x):=\lim _{n \rightarrow \infty} \frac{Q\left(2^{n} x\right)}{4^{n}}
$$

exists for each $x \in X$ and defines a unique quadratic mapping $R: X \rightarrow Y$ such that

$$
\mu_{Q(x)-R(x)}(t) \geq \Lambda_{x,-x, x}\left(\frac{(4-\alpha) t}{2}\right) .
$$


for all $x \in X$ and all $t>0$.

Corollary 3.4. Let $\theta \geq 0$ and let $p$ be a real number with $0<p<1$. Let $X$ be a normed vector space with norm $\|$.$\| . Let Q: X \rightarrow Y$ be a quadratic mapping satisfying

$$
\mu_{Q(z-x)+Q(z-y)-\frac{1}{2} Q(x-y)-2 Q\left(z-\frac{x+y}{2}\right)}(t) \geq \frac{t}{t+\theta\left(\|x\|^{p}+\|y\|^{p}+\|z\|^{p}\right)}
$$

for all $x, y, z \in X$ and all $t>0$. Then

$$
R(x):=\lim _{n \rightarrow \infty} \frac{Q\left(2^{n} x\right)}{4^{n}}
$$

exists for each $x \in X$ and defines an quadratic mapping $R: X \rightarrow Y$ such that

$$
\mu_{Q(x)-R(x)}(t) \geq \frac{\left(4-4^{p}\right) t}{\left(4-4^{p}\right) t+6 \theta\|x\|^{p}}
$$

Proof. The proof follows from Theorem (3.3) by taking

$$
\Lambda_{x, y, z}(t)=\frac{t}{t+\theta\left(\|x\|^{p}+\|y\|^{p}+\|z\|^{p}\right)}
$$

for all $x, y \in X$ and all $t>0$. Then we can choose $\alpha=4^{p}$ and we get the desired result.

\section{REFERENCES}

[1] P. W. Cholewa, Remarks on the stability of functional equations,Aequationes Math. 27 (1984), no. 1-2, 76-86.

[2] S. Czerwik, On the stability of the quadratic mapping in normed spaces, Abh. Math. Sem. Univ. Hamburg 62 (1992), 59-64.

[3] P. Găvruta, A generalization of the Hyers-Ulam-Rassias stability of approximately additive mappings, J. Math. Anal. Appl. 184 (1994), no. 3, 431-436.

[4] D. H. Hyers, On the stability of the linear functional equation, Proc. Nat. Acad. Sci. U. S. A. 27 (1941), 222-224.

[5] A. Najati and F. Moradlou, Hyers-Ulam-Rassias stability of the Apollonius yype quadratic mapping in non-Archimedean normed spaces, Tamsui Oxford J. Math. Sci., 24(4)(2008), 367-380.

[6] Th. M. Rassias, On the stability of the linear mapping in Banach spaces, Proc. Amer. Math. Soc. 72 (1978), no. 2, 297-300.

[7] R. Saadati and M. Vaezpour and Y.J. Cho, A note to paper "On the stability of cubic mappings and quartic mappings in random normed spaces, J. of Inequalities and Applications, Vol. 2009, Article ID 214530.

[8] B. Schewizer and A. Sklar, Probabilistic Metric Spaces, North-Holland Series in Probability and Applied Mathematics, North-Holland, New York, NY, USA, 1983.

[9] F. Skof, Local properties and approximation of operators, Rend. Sem. Mat. Fis. Milano 53 (1983), 113-129.

[10] S. M. Ulam, A collection of mathematical problems, Interscience Tracts in Pure and Applied Mathematics, no. 8 Interscience Publishers, New York-London, 1960.

1,2,3,4 Department of Mathematics, College of Science, Yasouj University, YaSOUJ 75914-353, IRAN

E-mail address: azadi@mail.yu.ac.ir 